\title{
Strain coupling in multiferroic phase transitions of samarium yttrium manganite $\mathrm{Sm}_{0.6} \mathrm{Y}_{0.4} \mathrm{MnO}_{3}$
}

\author{
Jason Schiemer, ${ }^{1, *}$ Daniel O’Flynn, ${ }^{2, \dagger}$ Geetha Balakrishnan, ${ }^{2}$ and Michael A. Carpenter ${ }^{1}$ \\ ${ }^{1}$ Department of Earth Sciences, University of Cambridge, Downing Street, Cambridge CB2 3EQ, United Kingdom \\ ${ }^{2}$ Department of Physics, University of Warwick, Coventry CV4 7AL, United Kingdom
}

(Received 30 May 2013; published 16 August 2013)

\begin{abstract}
$\mathrm{Sm}_{1-x} \mathrm{Y}_{x} \mathrm{MnO}_{3}(\mathrm{SYMx})$ is one of a class of multiferroic manganites that has seen significant recent interest due to the intimate connection between cycloidal magnetic order and ferroelectricity in these materials. SYM shows sequential transitions between paramagnetic, sinusoidally ordered antiferromagnetic and cycloidally ordered antiferromagnetic phases with decreasing temperature. As in the other members of the family, the magnetic spin cycloid induces ferroelectricity, although whether there is any elastic coupling involved in this process is not known. In this work, resonant ultrasound spectroscopy (RUS) is used to examine the stiffness and dissipation in SYM 0.4 as the magnetic transitions are traversed. It is found that there are only very small signatures of the transitions in the elastic properties of the material, indicating the weakness of the magnetoelastic (and electroelastic) coupling. The mechanical loss does show a significant decrease upon cooling below $T_{N 1}=\sim 50 \mathrm{~K}$, indicating the freezing of some loss mechanism near the temperature where magnetic order is achieved. The strain at these magnetic transitions in a related material, $\mathrm{Eu}_{1-x} \mathrm{Y}_{x} \mathrm{MnO}_{3}$, is examined from data published in the literature, and very low shear strain is observed, along with a more significant volume strain effect. This correlates well with the observations from RUS, as the peak frequencies are more sensitive to shear effects than bulk effects. These results suggest that the weak coupling of the magnetic transitions with shear may be a more general behavior in multiferroic perovskite-related manganites.
\end{abstract}

DOI: 10.1103/PhysRevB.88.054108

\section{INTRODUCTION}

Multiferroics are a class of materials that exhibit simultaneous ferroic order of at least two types, generally two or three of ferroelectric, (anti)ferromagnetic, and ferroelastic. In these systems, the various types of order present may or may not be coupled to one another. The relative behavior of these types of order is of key importance for device applications. In particular, strong magneto-electric coupling is ideal for many kinds of devices, including spintronics and memory applications, ${ }^{1,2}$ where an intimate connection between the magnetic and electrical state of the system delivers the effects required, for example, in electrically written, magnetically read nonvolatile memory elements. ${ }^{3}$ Both single phase and multiphase (composite) multiferroics have seen a significant degree of investigation. In the former, the role of strain in any magnetoelectrical behavior is often poorly understood, or totally uninvestigated, while in the latter, a strain mediated coupling from electrical order in one phase to magnetic order in the other creates the desired magnetoelectrical phenomena. ${ }^{4}$ Single-phase multiferroics can be broadly broken into two categories, namely "type-II" and "type-I"materials. The subject of this present work is $\mathrm{Sm}_{1-x} \mathrm{Y}_{x} \mathrm{MnO}_{3}$ (SYM x), specifically SYM 0.4, a recently discovered member of the type-II multiferroic rare earth manganite family $\left(R \mathrm{MnO}_{3}\right.$, with $R$ a rare earth ion).

Type-II magnetic systems show spin-driven ferroelectricity, ${ }^{5}$ and include, along with $R \mathrm{MnO}_{3}$, materials such as $\mathrm{CuFeO}_{2}$, which only exhibit this behavior under applied magnetic field. ${ }^{6}$ The spontaneous strain in most type-II materials at their magnetic transitions has seen little investigation, although significant magnetostriction has been reported in both $\mathrm{CuFeO}_{2}{ }^{6}$ and $\mathrm{SmMnO}_{3} .{ }^{7} \mathrm{CuFeO}_{2}$ exhibits a simultaneous structural and magnetic transition, which leads to significant changes in acoustic velocities, ${ }^{8}$ but the
PACS number(s): 77.80.-e, 75.85.+t, 75.80.+q, 62.40.+i possibility for this sort of behavior has not been examined in $R \mathrm{MnO}_{3}$. Many members of the $R \mathrm{MnO}_{3}$ family show type-II multiferroic behavior, with $\mathrm{Eu}_{1-x} \mathrm{Y}_{x} \mathrm{MnO}_{3}, \mathrm{DyMnO}_{3}$, $\mathrm{TbMnO}_{3},{ }^{8,9}$ and $\mathrm{SYM}^{10}$ exhibiting ferroelectricity driven by cycloidal magnetic ordering. ${ }^{11}$ These systems also show a magnetically driven polarization flop, which in SYM is from predominantly along the $c$ axis to predominantly along the $a$ axis under a magnetic field of $7 \mathrm{~T}$ along the $c$ axis. ${ }^{10}$ This field driven spin flop and intrinsic magnetoelectric coupling differentiate type-II multiferroic manganites from type-I systems. In type-I systems, the transition temperatures for magnetic and ferroelectric ordering are high, and in general unrelated to one another. The ferroelectric polarization is up to $10^{4}$ times larger than in type-II systems $\left(\sim 1 \times 10^{6} \mu \mathrm{C} / \mathrm{m}^{2}\right.$ in type-I bismuth ferrite, ${ }^{12} \mathrm{cf} .1 .5 \times 10^{2} \mu \mathrm{C} / \mathrm{m}^{2}$ in SYM $\left.0.4^{10}\right)$. Unfortunately, the associated strain is also large, creating a large potential barrier to polarization change. For example, bismuth ferrite shows an extremely high coercive field of $10-20 \mathrm{kV} / \mathrm{cm}$ in single crystals ${ }^{12}$ and up to $500 \mathrm{kV} / \mathrm{cm}$ in thin films. ${ }^{13}$ Additionally, in type-I systems, despite seemingly useful behaviors such as large polarization, any coupling between magnetic and electrical orders is weak due to the magnetic and electrical order parameters being decoupled. ${ }^{14}$

Among the type-II multiferroics, SYM itself was chosen as a target for a novel synthesis and investigation by design by O'Flynn et al. ${ }^{10}$ in an analogous way to previous investigations of $\mathrm{Eu}_{1-x} \mathrm{Y}_{x} \mathrm{MnO}_{3}$, whereby the $\mathrm{Y}$ atom is doped into the structure to partially replace the $A$ site ion, (in this case $\left.\mathrm{Sm}^{I I I}\right)$. This induces a distortion of the structure due to the difference in ionic size. The dopant concentration is chosen to bring this distortion into the range that has been determined to be required for the spin-driven ferroelectric phase to occur. This distortion is often quantified by the Mn-O-Mn (MOM) bond angle for reasons of magnetic exchange interaction ${ }^{15,16}$ 
and the key range of angles needed to create spin-driven ferroelectricity appears to be $\sim 144.5-145.6^{\circ} .{ }^{9}$ A systematic variation in the Néel temperatures has been observed with variation in this MOM angle. The Néel temperatures for other members of the $R \mathrm{MnO}_{3}$ family range from $\sim 47 \mathrm{~K}$ for Ho, with an MOM angle of $\sim 144^{\circ}$, to a minimum at $\sim 40 \mathrm{~K}$ for Dy, with a MOM angle of $\sim 144.7^{\circ}$ and then up to $\sim 85 \mathrm{~K}$ for $\mathrm{Nd}$ with a MOM angle of $\sim 150^{\circ} .{ }^{9}$ SYM 0.4, along with $\mathrm{TbMnO}_{3}$ and Eu doped $\mathrm{YMnO}_{3}$, exhibits a sinusoidally modulated antiferromagnetic phase below $T_{N 1}=50 \mathrm{~K}$ and a ferroelectric cycloidally modulated antiferromagnetic phase below $T_{N 2}=\sim 27 \mathrm{~K} .{ }^{10,17}$ The members of this family with perovskite $A$ site (rare earth site) magnetic moments show additional ordering at a lower temperature than the initial Néel temperature and the cycloidal ordering temperature (where present) and can show a different electrical polarization direction to those without magnetic $A$ site ions. ${ }^{18}$ In these materials, the initial electrical polarization is along the $c$ axis of the Pbnm structure rather than the $a$ axis as found in materials with a nonmagnetic $A$ site ion. Beyond this region, Eu doped $\mathrm{YMnO}_{3}$, for example, shows a change in spin cycloid to the $a-b$ plane and a change in polarization from the $c$ axis to the $a$ axis below $T_{N 3}=20 \mathrm{~K} .{ }^{17,19}$ As mentioned above, SYM 0.4 displays an electrical dipole along the $c$ axis, ${ }^{10}$ indicating that the $\mathrm{Sm}^{I I I}$ magnetic moments may be playing a significant part in the behavior of the material. This correlates with a phase transition observed at the proposed $T_{N 3}=\sim 4 \mathrm{~K}$, which is ascribed to ordering of the $\mathrm{Sm}^{I I I}$ magnetic moments. ${ }^{10}$

This paper seeks to examine the nature of any strain coupling at the low temperature transitions in SYM 0.4 using resonant ultrasound spectroscopy (RUS). RUS is sensitive to even small changes in strain, as the elastic constants measured in the experiment are essentially the susceptibility of the free energy with respect to strain ${ }^{20}$ and as such they vary much more strongly than the strains themselves. Strain coupling has previously been described as a possible requirement, at least in some systems, for multiferroic behavior. The theory is that the magnetic and electric order parameters are each coupled with strain, such that one order parameter interacts with the other via a common strain. ${ }^{21}$ Atomic shifts associated with the small polarization that results from the magnetic origin of the ferroelectric effect in type-II materials such as SYM must also be small. Therefore, at most a small strain is expected upon the switch from sinusoidal to cycloidal incommensurate magnetic ordering at $T_{N 2}$. As the $T_{N 1}$ antiferromagnetic transition intrinsically does not require atomic shifts, in contrast to the ferroelectric transition, it is likely that there will be no significant strain behavior observed at this temperature. Weakly strain coupled magnetic behavior of this type has previously been seen in $\mathrm{KMnF}_{3}{ }^{22}$ and $\mathrm{Pr} / \mathrm{Ca}^{23}$ and $\mathrm{Pr} / \mathrm{Sr}^{24}$ manganite solid solutions. If the strain is indeed small at these transitions, it will reflect the truly different nature of these type-II multiferroics when compared to the type-I systems most investigated in the past.

\section{EXPERIMENTAL DETAILS}

A piece of the $\mathrm{Sm}_{0.6} \mathrm{Y}_{0.4} \mathrm{MnO}_{3}$ single crystal previously investigated by O'Flynn et al. ${ }^{10}$ was used in this work. The single crystal boule that the sample was taken from was

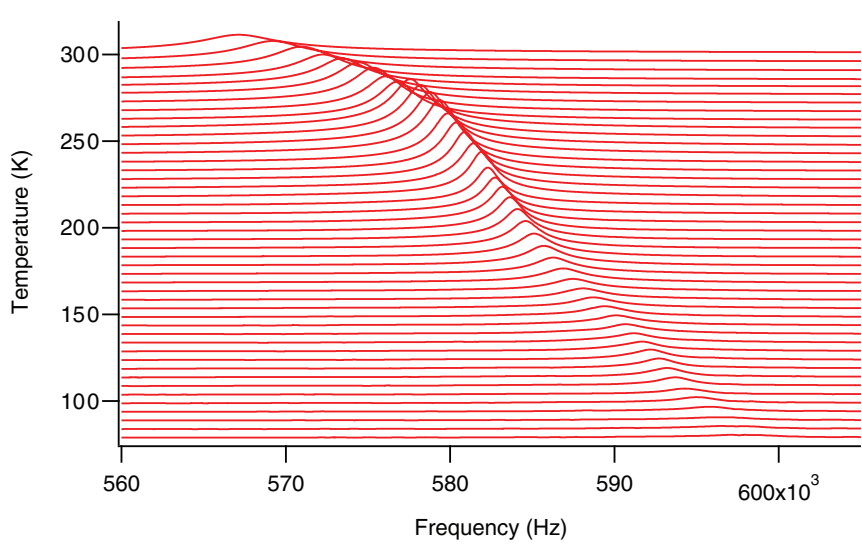

FIG. 1. (Color online) Stack of sections of RUS spectra, collected in a heating sequence, showing a single peak between $100 \mathrm{~K}$ and room temperature.

synthesized by the floating zone crystallization technique at the University of Warwick, as described in Ref. 10. An irregularly shaped piece was used (with dimensions of $\sim 3.9 \times 1.75 \times 1.6 \mathrm{~mm}$ and mass of $0.1041 \mathrm{~g}$ ). RUS spectra were collected in situ at both low and high temperature. The low temperature sample environment is in an Orange He flow cryostat, and DRS $\mathrm{M}^{3}$ ODULUS II electronics are used for data collection, as described by McKnight et al ${ }^{25}$ The data collected are routinely transferred to the software package IGOR PRO (Wavemetrics) for analysis. Selected peaks, and their trends with temperature, were analyzed by fitting with an asymmetric Lorentzian peak function. This fitting determines the peak frequency, $f$, and the peak width at half maximum height, $\Delta f$. This information is converted to a measure of the internal friction through the mechanical quality factor $Q$, defined as $Q=f / \Delta f$.

\section{RESULTS}

Two stacks of RUS spectra are shown from $300 \mathrm{~K}$ to $100 \mathrm{~K}$ in Fig. 1 and from $100 \mathrm{~K}$ to $5 \mathrm{~K}$ in Fig. 2, while data analyzed for $f^{2}$ for a number of peaks are presented in Fig. 3 and Fig. 4. Inverse quality factor (mechanical loss, $Q^{-1}$ ) data are also presented in Fig. 3. These data were collected between 50 and

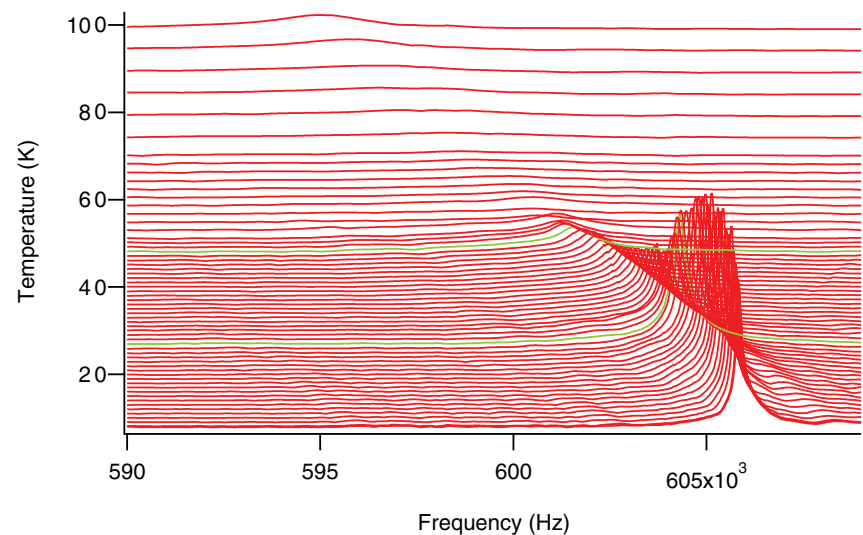

FIG. 2. (Color online) Stack of sections of RUS spectra, collected in a heating sequence at low temperature, showing a single peak below $100 \mathrm{~K}$. Green traces indicate the expected transition temperatures. 


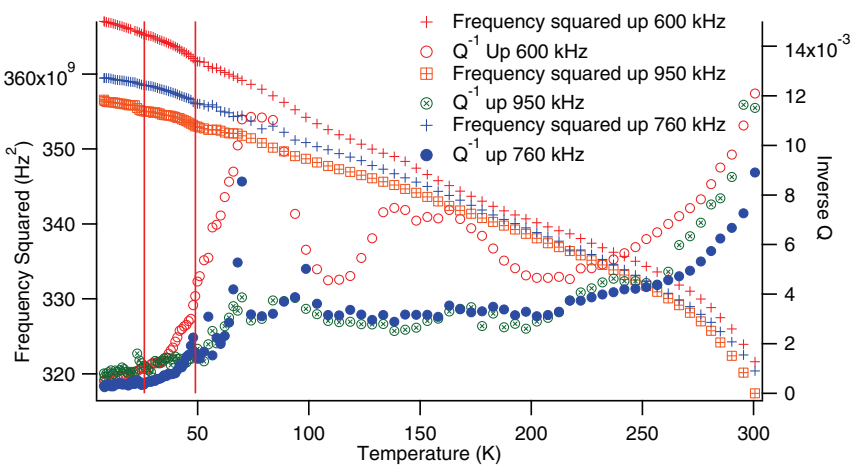

FIG. 3. (Color online) Squared frequency and inverse quality factor, $Q^{-1}$, for two frequencies in SYM 0.4. The overall trend of softening in squared frequency shows slight deviations at $T_{N 1}$ and $T_{N 2}$, marked with red lines, as well as extra softening observed above $250 \mathrm{~K}$. There is a clear decrease in $Q^{-1}$ below $\sim 60-70 \mathrm{~K}$ and a significant increase above $\sim 250 \mathrm{~K}$. Peaks occur at $70 \mathrm{~K}, 100 \mathrm{~K}$, and $150 \mathrm{~K}$ in $Q^{-1}$.

$1300 \mathrm{kHz}$, with 65000 data points per spectrum. Temperature steps used were $30 \mathrm{~K}$ in cooling and $5 \mathrm{~K}$ in heating. A dwell period of $20 \mathrm{~min}$ was used at each temperature to ensure thermal equilibration. The left axis is, in fact, amplitude in volts, but an offset to the spectra has been applied such that the intercept of the spectrum denotes the temperature at which it was collected. The raw spectra show clear anomalies with changes in temperature, as well as more subtle features (Fig. 1 and Fig. 2). The more detailed analysis provided in Fig. 3 and Fig. 4 also show these changes in elastic and anelastic behavior, as well as some that are more subtle than those readily observed in the raw data. These features will be described in sequence of decreasing temperature. Note that the squared peak frequency, as shown in Fig. 3 and Fig. 4, is proportional to the stiffness of the sample and that the normal modes of the sample are dominated by shearing, particularly at low frequencies. The overall pattern is of stiffening with decreasing temperature (clearly seen in Fig. 1 and Fig. 2), as normally expected for a crystalline material, but superimposed on this are some interesting discrepancies. The peak frequencies show a marked

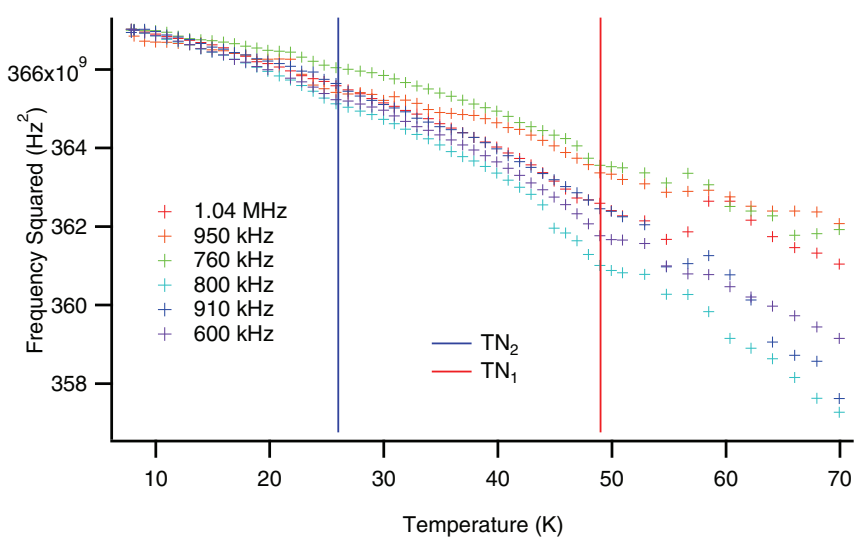

FIG. 4. (Color online) Analyzed squared peak frequencies $\left(f^{2}\right)$ from RUS spectra at low temperature, with absolute values rescaled to those of the peak at $\sim 600 \mathrm{kHz}$. The expected transition temperatures are shown by vertical lines. softening near room temperature, combined with an increase in $Q^{-1}$ as temperature is increased (Fig. 3). Figure 3 shows that there is a definite trend to lower $Q^{-1}$ below about $60 \mathrm{~K}$ and to higher $Q^{-1}$ as the temperature is raised from $250 \mathrm{~K}$ to room temperature. Very slight anomalies in $f^{2}$ are observed near the two previously reported magnetic phase transitions, at $T_{N 1}(\sim 49 \mathrm{~K})$ and $T_{N 2}(\sim 26 \mathrm{~K})$, as can primarily be seen in Fig. 4; both of these temperatures correlate with a minimum in the frequency all of the peaks followed in detail and are in a region of decreasing $Q^{-1}$ (Fig. 3). The data for $Q^{-1}$ additionally show Debye-like peaks between $\sim 70$ and $\sim 200$ $\mathrm{K}$, although this loss behavior between 100 and $200 \mathrm{~K}$ is only seen in data from one resonance and may be due to an artifact associated with, for example, a microcrack in the sample.

\section{DISCUSSION}

In the RUS data for SYM 0.4, it is expected that the resonant frequencies at low frequency are dominated by shear rather than the bulk modulus, and, as such, the behavior of the peaks analyzed should be dominated by combinations of shear elastic constants. Due to having an irregularly shaped piece of single crystal, values for individual elastic constants cannot be uniquely determined from the peak frequencies. Tracking the behavior of resonant peaks nevertheless reveals the general pattern of evolution of elastic constants that relate to shear. The origin of elastic and anelastic anomalies is related in some way to strain, which is therefore considered first.

In perovskite related materials, a common way to predict the source of static strains expected away from the ideal structure is to use the tolerance factor. The tolerance factor, $t$, is given by $t=\frac{r_{X}+r_{A}}{\sqrt{2}\left(r_{X}+r_{B}\right)}$, where $r_{X}$ is the radius of the anion, $r_{A}$ is the radius of the cuboctahedrally coordinated " $a$ "site cation, and $r_{B}$ is the radius of the octahedrally coordinated " $B$ "site cation. For SYM 0.4, this is equal to $0.903 \pm \sim 0.002$, which is on the cusp of cubic stability from classic perovskite theory. ${ }^{26}$ This calculation uses Shannon's ionic radii ${ }^{27}$ and an estimate of the ionic radius for XII coordinate $\mathrm{Y}$ as 1.3 times the radius of VI coordinate $\mathrm{Y}$, based on an examination of other rare earth ions where both VI and XII are given by Shannon. Pure $\mathrm{YMnO}_{3}$ has a tolerance factor of 0.89 , while pure $\mathrm{SM}$ has a tolerance factor of 0.912. Despite the fact that SM has a tolerance factor that from a purely ionic point of view puts it on the boundary of a region where a cubic, untilted, perovskite would be expected, ${ }^{26}$ the trends from neighboring rare earths suggested large tilts of the octahedra, with an orthorhombic Pbnm space group. ${ }^{15,28}$ This was confirmed by O'Flynn ${ }^{29}$ using isotope enriched $\mathrm{Sm}$ to decrease the incoherent scattering cross section in neutrons, and an Mn-O-Mn bond angle of $147.82^{\circ}$ was observed. YM has a tolerance factor that suggests tilting, and it is in the same Pbnm space group. ${ }^{30}$ This is repeated in the SYM solid solution phase, as it is not unexpected from its constituent members, and SYM 0.4 shows a highly distorted Mn-O-Mn bond angle of $145.79^{\circ} .{ }^{10}$ The origin of this large tilt and resultant shearing of the octahedra is due mostly to a cooperative Jahn Teller distortion of the $\mathrm{Mn}^{I I I}$ octahedra. ${ }^{31}$ In these manganites, there are in fact roughly equal components to the observed unit cell distortion from Jahn-Teller distortion of the octahedra and tilting of the octahedra. ${ }^{15}$ 
Despite the heavily sheared nature of the underlying structure in these perovskite manganites, the development of strains associated specifically with the low temperature magnetic/multiferroic phase transitions of interest technologically has not previously been explored in the literature. If the changes in magnetic ordering at the Néel temperatures were coupled with any significant shear strains, it would be expected that they would give rise to discontinuous softening in the elastic constants of the material, due to coupling terms of the form $\lambda e m^{2}$, where $e$ is a shear strain, $m$ is the magnetic order parameter, and the coupling coefficient, $\lambda$, defines the strength of coupling. ${ }^{20,32}$ Biquadratic coupling terms of the form $\lambda e^{2} m^{2}$ are always allowed and, when $\lambda$ is small, their influence may be seen as stiffening (or softening, depending on the sign of $\lambda$ ) in proportion to the square of the order parameter. In order to investigate whether there is significant shear strain due to coupling of the type $\lambda \mathrm{em}^{2}$, we analyze here the strain behavior of the related and very closely analogous material $\mathrm{Eu}_{0.6} \mathrm{Y}_{0.4} \mathrm{MnO}_{3}$ (EYM 0.4), which has the same sequence of magnetic transitions at $T_{N 1} \approx 49 \mathrm{~K}, T_{N 2} \approx 28 \mathrm{~K}$, and $T_{N 3} \approx 20 \mathrm{~K} .{ }^{17,33}$

The strains in EYM 0.4 can be calculated from the lattice parameter data of Moreira et al., ${ }^{33}$ using the strain-lattice parameter statements in McKnight et al. ${ }^{34}$ The description of shear strains in the Pnma structure (which is obtained from the Pbnm setting by a transformation $a \rightarrow c, b \rightarrow a$, and $c \rightarrow b)$ are given by the following relationships: $e_{t x}=\frac{2 e_{1}-e_{2}-e_{3}}{\sqrt{3}}$, $e_{4}=\frac{\frac{a}{\sqrt{2}}-a_{0}}{a_{0}}-\frac{\frac{c}{\sqrt{2}}-a_{0}}{a_{0}}$, and $e_{5}=e_{6}=0$, where $e_{1}=\frac{\frac{b}{\sqrt{2}}-a_{0}}{a_{0}}$ and $e_{2}+e_{3}=\frac{\frac{a}{\sqrt{2}}-a_{0}}{a_{0}}+\frac{\frac{c}{\sqrt{2}}-a_{0}}{a_{0}}$. Here, $a_{0}$ is the reference parameter of the parent cubic phase extrapolated into the stability field of the orthorhombic phase but, because there are no high temperature data to allow this extrapolation, it can be approximated as $a_{0} \approx\left(\frac{V}{4}\right)^{\frac{1}{3}}$ for calculation of shear strains. $V$ is the unit cell volume of the Pnma structure. Values of $e_{1}$ and $e_{2}+e_{3}$ and the shear strains $e_{4}$ and $e_{t x}$ obtained in this way are given in Fig. 5.

The first point to note in the data (Fig. 5) is the very large absolute values of strains involved, on the order of $7 \%-10 \%$ for shear strains and $\sim 4 \%$ for linear strains. These are due to Jahn Teller effects and octahedral tilting, but the shear strains vary negligibly through the region of the Néel temperatures [below the level of error in the measurement, Figs. 5(b) and 5(c)]. As previously reported [and visible in the data reproduced in Figs. 6(a) and 6(b)] there is a measurable volume anomaly at low temperature in the data of Moreira et al. ${ }^{33}$ for EYM 0.4. This has been determined as a volume strain here by first fitting an expression of the form $V=V_{1}+V_{2} \Theta_{o s} \operatorname{coth} \frac{\Theta_{o s}}{T}$ to the data above $40 \mathrm{~K}$, giving $V_{1}=$ $228.5, V_{2}=0.0056125, \Theta_{o s}=204.83$, and extrapolating this to $0 \mathrm{~K}$ [as described by McKnight et al., ${ }^{34}$ following Salje et al., ${ }^{35}$ and shown in Fig. 6(a)]. The volume strain with respect to the parent Pnma structure is then $V_{s}=\frac{V^{\prime}-V}{V}$, where $V^{\prime}$ is the unit cell volume below $\sim 70 \mathrm{~K}$ and shown in Fig. 6(b). By comparison with Figs. 5(a) and 5(b), it is apparent that there is a nearly isotropic contraction along the crystallographic $b$ axis (Pnma) and within the $a$-c plane $\left(V_{s} \approx e_{1}+e_{2}+e_{3}\right.$, change in $e_{2}+e_{3} \sim 2 \times$ change in $\left.e_{1}\right) . V_{s}$ has a clear minimum between $T_{N 1}$ and $T_{N 2}$, and possible breaks in slope at each transition temperature.
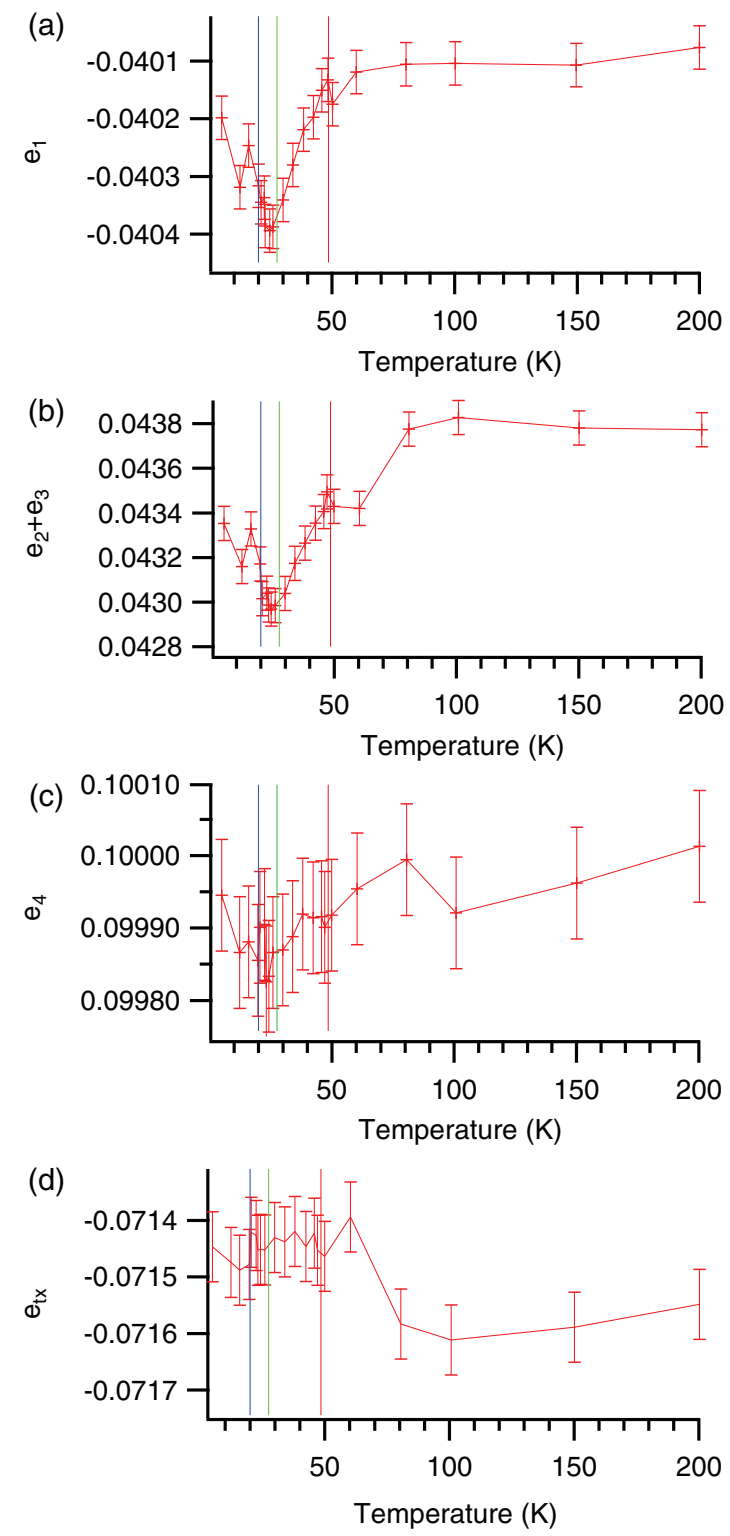

FIG. 5. (Color online) (a),(b) Volume strains $e_{1}$ and $e_{2}+e_{3}$ and (c),(d) shear strains $e_{4}$ and $e_{t x}$, versus temperature for $\mathrm{Eu}_{0.6} \mathrm{Y}_{0.4} \mathrm{MnO}_{3}$, as calculated from data in Moreira et al., ${ }^{33}$ with the magnetic phase transitions indicated by vertical lines.

Both $V_{s}$ and $e_{t x}$ also show a deviation at approximately 75 K [Fig. 6(b) and Fig. 5(d)]. This does not coincide with any known transitions in EYM 0.4 and is most likely due to precursor effects, i.e., dynamic or static clustering in a temperature interval of $\sim 25 \mathrm{~K}$ above the first magnetic transition point. The geometry of this additional anomaly is a contraction in the $a$-c plane $\left(e_{2}+e_{3}\right)$, with no equivalent change in the $b$ direction $\left(e_{1}\right)$, which is then an effective shear strain, $e_{t x}$, which has positive sign. $T_{N 1}$ corresponds to the paramagnetic to sinusoidal antiferromagnetic transition which then transforms to cycloidal at $T_{N 2}$. The direction of the propagation of the sinusoid and cycloid are along $a$ in both SYM 0.4 and EYM 0.4 in the Pnma setting, and the ferroelectric polarization direction due to the cycloid is parallel to $b$ in both materials. ${ }^{10,17,36}$ From this geometry it is 

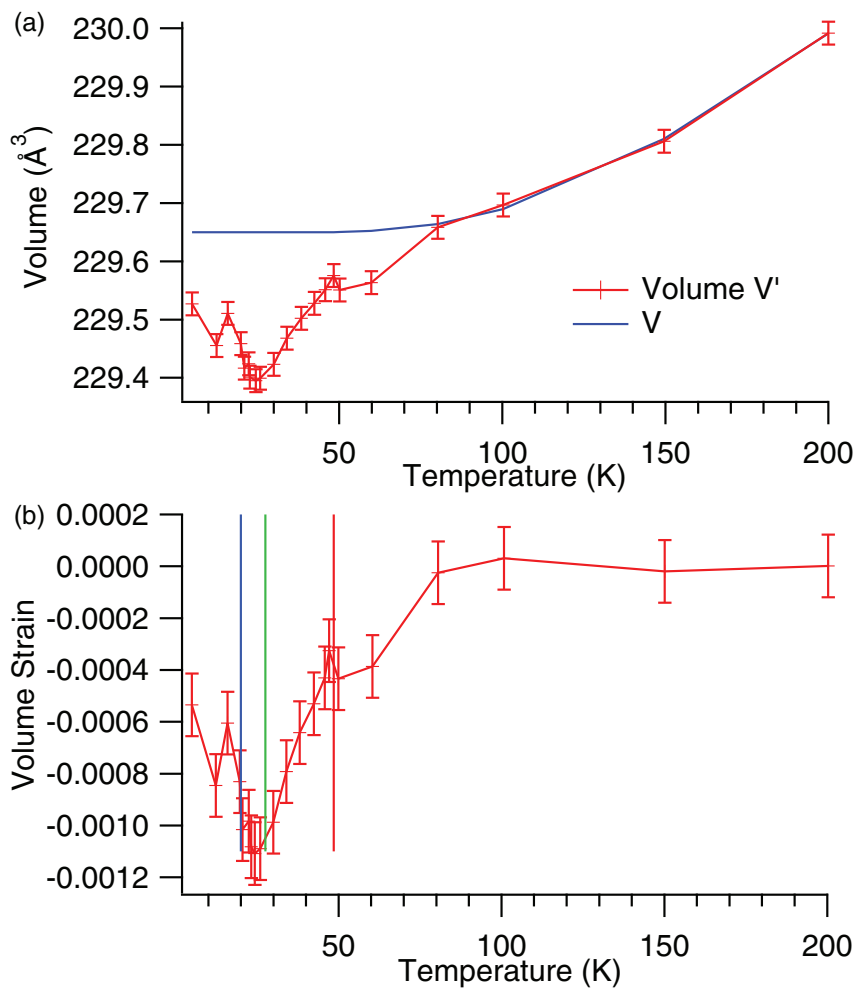

FIG. 6. (Color online) (a) Volume versus temperature for $\mathrm{Eu}_{0.6} \mathrm{Y}_{0.4} \mathrm{MnO}_{3}$, as calculated from data in Moreira et al., ${ }^{33}$ fitted with the function $V=V_{1}+V_{2} \Theta_{o s} \operatorname{coth} \frac{\Theta_{o s}}{T}$. (b) Volume strain versus temperature for $\mathrm{Eu}_{0.6} \mathrm{Y}_{0.4} \mathrm{MnO}_{3}$, as calculated from data in Moreira et al., ${ }^{33}$ with the magnetic phase transitions indicated by vertical lines.

apparent that the anomalous strain appearing at $\sim 75 \mathrm{~K}$ has its unique axis aligned parallel to the ferroelectric polarization of the cycloid. This potentially implies that local structure in the precursor interval involves local ferroelectric dipoles. An additional consequence of this anomaly is that the precursor effect is necessarily coupled to octahedral tilting and shearing through the common $e_{t x}$ strain, but indirect coupling by this mechanism would be very weak given the small anomaly in $e_{t x}$ associated with the magnetism.

The RUS data collected for this work on SYM 0.4 show a peak in $Q^{-1}$ and a possible break in slope of $f^{2}$ at around $\sim 70 \mathrm{~K}$ (Fig. 3). As with EYM 0.4, there are no reports in the literature of any magnetic behavior at this temperature, but the most likely cause is again the onset of some premonitory short range order. This will require verification by other experimental methods but it could potentially be a general pattern of behavior for these types of multiferroic materials. Near both magnetic transitions, $T_{N 1}$ and $T_{N 2}$, only very small anomalies are observed in the frequencies of the analyzed peaks (Fig. 4). These small anomalies do not show an obvious discontinuous drop in the shear elastic constants, consistent with the expectation from EYM 0.4 that coupling with shear strains $e_{4}$ and $e_{t x}$, defined with respect to the parent cubic structure, is weak/negligible, i.e., $\lambda$ in the $\lambda \mathrm{em}^{2}$ linear quadratic coupling term is small. Below $T_{N 1}$, though, there does appear to be stiffening behavior, which is likely to be a consequence of $\lambda e^{2} m^{2}$ biquadratic coupling terms, as has been observed also for several other materials with weak magnetoelastic coupling. ${ }^{37-39}$

Despite the small signature in the modulus near $T_{N 1}$ and $T_{N 2}$ there is a systematic decrease in $Q^{-1}$ with falling temperature (Fig. 3). This pattern is the reverse of what is observed for most phase transitions, as intrinsic acoustic losses at RUS frequencies are typically low in the stability field of the paraphase, while microstructure dynamics are responsible for variations in loss in the low symmetry phase. The pattern of low losses below the transition point and increasing loss above it has been observed in a few particular cases, however. For example, the mineral lawsonite, $\mathrm{CaAl}_{2} \mathrm{Si}_{2} \mathrm{O}_{7}(\mathrm{OH})_{2} \cdot \mathrm{H}_{2} \mathrm{O}$, has two transitions for which the primary driving order parameter relates to changes in hydrogen bonding. ${ }^{40,41}$ High values of $Q^{-1}$ above the transition temperatures can be understood in terms of coupling between strain and local dynamical disordering of the protons between symmetry-related positions. $Q^{-1}$ diminishes through the ordering transition points and becomes small once the protons become fixed in their low symmetry positions..$^{25} \mathrm{~A}$ similar argument has been applied to acoustic loss through a ferroelectric transition in a metalorganic framework phase, $\left[\left(\mathrm{CH}_{3}\right)_{2} \mathrm{NH}_{2}\right] \mathrm{Co}(\mathrm{HCOO})_{3}$, which also displays a peak in the acoustic loss $\sim 50 \mathrm{~K}$ above the transition point. ${ }^{37} \mathrm{~A}$ very closely similar pattern has also been observed through the ferroelastic (hexagonal $\rightarrow$ monoclinic) transition in $\mathrm{SrAl}_{2} \mathrm{O}_{4}: \mathrm{Eu}^{42}$ Perhaps the common feature of these is order/disorder character for the transitions, with dynamical disordering/clustering above the transition point. For SYM 0.4, the argument would be that there is some coupling of the electric dipole moments (on the basis of the strain orientation shown by EYM 0.4) to local strain above $T_{N}$ with frequencies not so far from those of the RUS experiment. On this basis, the onset of clustering would be identified with the peaks in $Q^{-1}$ above $T_{N}$ and the lowering of $Q^{-1}$ through $T_{N}$ would be attributed to diminution of any dynamic disorder. There is, as yet, no indication as to what additional disordering might be responsible for the increase in acoustic loss and associated elastic softening near room temperature.

\section{CONCLUSIONS}

The elastic behavior of SYM 0.4 at its Néel temperatures shows only a very slight signature for the two antiferromagnetic transitions, $T_{N 1}(\sim 49 \mathrm{~K})$ and $T_{N 2}(\sim 26 \mathrm{~K})$. Spontaneous strain in the related material EYM 0.4 shows large absolute shear strains due to Jahn-Teller distortions and octahedral tilting on which subtle variations associated with the magnetic ordering are superimposed. Thus it is clear that coupling of the magnetic order parameter with strain is only weak. However, anomalies in the acoustic loss $\sim 20-30$ $\mathrm{K}$ above $T_{N 1}$ appear to be indicative of the development of precursor fluctuations/clustering involving local electric dipoles. Dynamical disorder coupled with local strain above $T_{N 1}$ then diminishes through the transition temperatures. The small coupling between shear strain and magnetic transitions in SYM 0.4 (and EYM 0.4) begs the question of whether this is coincidental, or whether the magnetically driven ferroelectricity requires transitions that do not couple strongly with shear. 


\section{ACKNOWLEDGMENTS}

RUS facilities in Cambridge were established through support from the NERC (NE/B505738/1) to M.A.C.; G.B. acknowledges financial support through EPSRC, UK, Grant No. EP/I007210/1.

\section{AUTHOR CONTRIBUTIONS}

J.S. carried out the experiments, analyzed the data, and wrote the manuscript. M.A.C. designed the experiment and wrote the manuscript. D.O. and G.B. synthesized and provided the previously characterized samples. All authors discussed the results. *jas263@cam.ac.uk

${ }^{\dagger}$ Also at Department of Medical Physics and Bioengineering, University College London, Malet Place Engineering Building, Gower Street, London, WC1E6BT, UK.

${ }^{1}$ C. Binek and B. Doudin, J. Phys. Condens. Matter 17, L39 (2005).

${ }^{2}$ M. Gajek, M. Bibes, S. Fusil, K. Bouzehouane, J. Fontcuberta, A. Barthlmy, and A. Fert, Nat. Mater. 6, 296 (2007).

${ }^{3}$ J. F. Scott, Nat. Mater. 6, 256 (2007).

${ }^{4}$ C.-W. Nan, M. I. Bichurin, S. Dong, D. Viehland, and G. Srinivasan, J. Appl. Phys. 103, 031101 (2008).

${ }^{5}$ S.-W. Cheong and M. Mostovoy, Nat. Mater. 6, 13 (2007).

${ }^{6}$ T. Kimura, J. C. Lashley, and A. P. Ramirez, Phys. Rev. B 73, 220401 (2006).

${ }^{7}$ J.-S. Jung, A. Iyama, H. Nakamura, Y. Wakabayashi, and T. Kimura, Phys. Rev. B 85, 174414 (2012).

${ }^{8}$ G. Quirion, M. J. Tagore, M. L. Plumer, and O. A. Petrenko, Phys. Rev. B 77, 094111 (2008).

${ }^{9}$ T. Goto, T. Kimura, G. Lawes, A. P. Ramirez, and Y. Tokura, Phys. Rev. Lett. 92, 257201 (2004).

${ }^{10}$ D. O'Flynn, C. V. Tomy, M. R. Lees, A. Daoud-Aladine, and G. Balakrishnan, Phys. Rev. B 83, 174426 (2011).

${ }^{11}$ M. Mostovoy, Phys. Rev. Lett. 96, 067601 (2006).

${ }^{12}$ D. L. D. C. A. Forget and M. Viret, Appl. Phys. Lett. 91, 022907 (2007).

${ }^{13}$ H. Liu and X. Wang, J. Sol-Gel Sci. Technol. 47, 154 (2008).

${ }^{14}$ C. Ederer and N. A. Spaldin, Phys. Rev. B 71, 060401 (2005).

${ }^{15}$ J. A. Alonso, M. J. Martínez-Lope, M. T. Casais, and M. T. Fernández-Díaz, Inorg. Chem. 39, 917 (2000).

${ }^{16}$ V. Laukhin, J. Fontcuberta, J. L. García-Muñoz, and X. Obradors, Phys. Rev. B 56, R10009 (1997).

${ }^{17}$ J. Hemberger, F. Schrettle, A. Pimenov, P. Lunkenheimer, V. Y. Ivanov, A. A. Mukhin, A. M. Balbashov, and A. Loidl, Phys. Rev. B 75, 035118 (2007).

${ }^{18}$ R. Feyerherm, E. Dudzik, A. U. B. Wolter, S. Valencia, O. Prokhnenko, A. Maljuk, S. Landsgesell, N. Aliouane, L. Bouchenoire, S. Brown et al., Phys. Rev. B 79, 134426 (2009).

${ }^{19}$ K. Noda, M. Akaki, T. Kikuchi, D. Akahoshi, and H. Kuwahara, J. Appl. Phys. 99, 08S905 (2006).

${ }^{20}$ M. A. Carpenter and E. K. H. Salje, Eur. J. Mineral. 10, 693 (1998).

${ }^{21}$ L. Oravova, Z. Zhang, N. Church, R. J. Harrison, C. J. Howard, and M. A. Carpenter, J. Phys. Condens. Matter 25, 116006 (2013).
${ }^{22}$ M. A. Carpenter, E. K. H. Salje, and C. J. Howard, Phys. Rev. B 85, 224430 (2012).

${ }^{23}$ E. K. H. Salje and M. A. Carpenter, J. Phys. Condens. Matter 23, 245401 (2011).

${ }^{24}$ T.-Y. Tan, B. J. Kennedy, Q. Zhou, C. D. Ling, W. Miiller, C. J. Howard, M. A. Carpenter, and K. S. Knight, Phys. Rev. B 85, 104107 (2012).

${ }^{25}$ R. E. McKnight, M. A. Carpenter, T. W. Darling, A. Buckley, and P. A. Taylor, Am. Mineral. 92, 1665 (2007).

${ }^{26} \mathrm{M}$. Johnsson and P. Lemmens, Crystallography and Chemistry of Perovskites (John Wiley \& Sons, Ltd., New York, 2007).

${ }^{27}$ R. D. Shannon, Acta Crystallogr. Sect. A 32, 751 (1976).

${ }^{28}$ L. Martín-Carrón, A. de Andrés, M. Martínez-Lope, M. Casais, and J. Alonso, J. Alloys Compd. 323-324, 494 (2001); Proceedings of the 4th International Conference on f-Elements, URL http://www. sciencedirect.com/science/article/pii/S0925838801010477.

${ }^{29}$ D. T. O'Flynn, Ph.D. thesis, The University of Warwick, 2010.

${ }^{30}$ A. Muñoz, J. A. Alonso, M. T. Casais, M. J. Martínez-Lope, J. L. Martínez, and M. T. Fernández-Díaz, J. Phys. Condens. Matter 14, 3285 (2002).

${ }^{31}$ M. W. Lufaso and P. M. Woodward, Acta Crystallogr. Sect. B 60, 10 (2004).

${ }^{32}$ W. Rehwald, Adv. Phys. 22, 721 (1973).

${ }^{33}$ J. Agostinho Moreira, A. Almeida, W. S. Ferreira, J. P. Araújo, A. M. Pereira, M. R. Chaves, M. M. R. Costa, V. A. Khomchenko, J. Kreisel, D. Chernyshov et al., Phys. Rev. B 82, 094418 (2010).

${ }^{34}$ R. E. A. McKnight, C. J. Howard, and M. A. Carpenter, J. Phys. Condens. Matter 21, 015901 (2009).

${ }^{35}$ E. Salje, B. Wruck, and S. Marais, Ferroelectrics 124, 185 (1991).

${ }^{36}$ H. Murakawa, Y. Onose, F. Kagawa, S. Ishiwata, Y. Kaneko, and Y. Tokura, Phys. Rev. Lett. 101, 197207 (2008).

${ }^{37}$ R. I. Thomson, P. Jain, A. K. Cheetham, and M. A. Carpenter, Phys. Rev. B 86, 214304 (2012).

${ }^{38}$ R. I. Thomson, J. M. Rawson, A. Goeta, M. R. Probert, J. A. Coome, T. K. Hoang, and M. A. Carpenter, Mater. Chem. Phys. 139, 34 (2013).

${ }^{39}$ R. I. Thomson, E. S. L. Wright, J. M. Rawson, C. J. Howard, and M. A. Carpenter, Phys. Rev. B 84, 104450 (2011).

${ }^{40}$ E. Libowitzky and T. Armbruster, Am. Mineral. 80, 1277 (1995).

${ }^{41}$ E. Libowitzky and G. R. Rossman, Am. Mineral. 81, 1080 (1996).

${ }^{42}$ M. A. Carpenter, C. J. Howard, M. J. Andrew, R. E. A. McKnight, Y. Liu, and R. L. Withers, J. Appl. Phys. 107, 013505 (2010). 